\title{
Widespread pyocyanin over-production among isolates of a cystic fibrosis epidemic strain
} Joanne L Fothergill ${ }^{1}$, Stavroula Panagea ${ }^{1}$, Charles A Hart ${ }^{1}$,
Martin J Walshaw ${ }^{2}$, Tyrone L Pitt ${ }^{3}$ and Craig Winstanley*1

Address: ${ }^{1}$ Division of Medical Microbiology and Genitourinary Medicine, University of Liverpool, Liverpool L69 3GA, UK, ${ }^{2}$ Regional Adult Cystic Fibrosis Unit, Cardiothoracic Centre, Liverpool L14 3PE, UK and ${ }^{3}$ Laboratory of HealthCare Associated Infection, Health Protection Agency, 61 Colindale Avenue, London, NW9 5HT, UK

Email: Joanne L Fothergill - j.fothergill@liv.ac.uk; Stavroula Panagea - vickypanagea@doctors.org.uk; Charles A Hart - cahmm@liv.ac.uk; Martin J Walshaw - mwalshaw@doctors.org.uk; Tyrone L Pitt - tyrone.pitt@hpa.org.uk; Craig Winstanley* - c.winstanley@liv.ac.uk

* Corresponding author

Published: 23 May 2007

BMC Microbiology 2007, 7:45 doi:10.1 |86/|47|-2/80-7-45
Received: II January 2007

Accepted: 23 May 2007

This article is available from: http://www.biomedcentral.com/I47I-2/80/7/45

(c) 2007 Fothergill et al; licensee BioMed Central Ltd.

This is an Open Access article distributed under the terms of the Creative Commons Attribution License (http://creativecommons.org/licenses/by/2.0), which permits unrestricted use, distribution, and reproduction in any medium, provided the original work is properly cited.

\begin{abstract}
Background: Some isolates of the Liverpool cystic fibrosis epidemic strain of Pseudomonas aeruginosa exhibit an unusual virulence-related phenotype, characterized by over-production of quorum sensing-regulated exoproducts such as pyocyanin and Las A protease. Our aim was to determine the prevalence of this unusual phenotype amongst isolates of the epidemic strain, and to study other intraclonal phenotypic and genotypic variations.

Results: The unusual phenotype was detected in at least one epidemic strain isolate from the majority of cystic fibrosis patients tested, and can be retained for up to seven years during chronic infection. Multiple sequential isolates of the epidemic strain taken from six patients over a period of up to nine years exhibited a wide range of phenotypes, including different antimicrobial susceptibilities. Our data suggest that each sputum sample contains a mixture of phenotypes and genotypes within the epidemic strain population, including within colony morphotypes. Many isolates exhibit premature (during early rather than late exponential growth) and over-production of pyocyanin, which has a number of toxic effects directly relevant to cystic fibrosis.
\end{abstract}

Conclusion: The widespread occurrence of this unusual phenotype suggests that it may play an important role in the success of the epidemic strain.

\section{Background}

With a carrier rate of 1 in 25 and an incidence of 1 in 2,500 live births, cystic fibrosis (CF) is the most common life-threatening, recessively inherited disease amongst Caucasians. During chronic pulmonary infection, CF patients suffer decline in lung function associated with periodic exacerbations triggered by bacterial products.
The most widespread CF pathogen is Pseudomonas aeruginosa, which causes infections that, once established, are impossible to eradicate.

The blue, redox-active phenazine pyocyanin is secreted by $P$. aeruginosa under the control of its cell-density-dependent quorum sensing (QS) system [1]. Pyocyanin and its precursor phenazine-1-carboxylic acid (PCA) have a number of toxic effects relevant to CF $[2,3]$. Pyocyanin 
and PCA inhibit the beating of human respiratory cilia in vitro $[4,5]$, and alter expression of immunomodulatory proteins by human airway epithelial cells [6]. For example, increases in IL-8 and intercellular adhesion molecule1 (ICAM-1) expression by oxidant-dependent mechanisms have been demonstrated in vitro and in vivo [6,7]. It has also been shown that pyocyanin production by $P$. aeruginosa induces neutrophil apoptosis and impairs neutrophil-mediated host defenses in vitro [8] and in vivo [9]. Furthermore, Kong et al. [10] have demonstrated that physiologically relevant concentrations of pyocyanin inactivate the airway vacuolar ATPase, resulting in reduced expression of $\mathrm{CF}$ transmembrane conductance regulator (CFTR) in cultured lung and nasal epithelial cells.

In 1996 Cheng et al. [11] used molecular typing to demonstrate the spread of a drug-resistant strain of $P$. aeruginosa [named the Liverpool Epidemic Strain (LES)] amongst patients in a children's CF centre in Liverpool, UK. Analysis of post-2000 patient samples identified the LES in $79 \%$ of $80 \mathrm{P}$. aeruginosa-colonised CF patients in the Liverpool adult CF centre, confirming the spread and longevity of LES infections [12]. Furthermore, a survey of 31 CF centres in England and Wales, involving the analysis of more than 1200 isolates of $P$. aeruginosa, identified the LES as the most common clone, being present in $48 \%$ of CF centres and accounting for $11 \%$ of the isolates [13].

In addition to its transmissibility, the LES appears to be more aggressive than other $P$. aeruginosa strains. It has been shown to replace previously established strains of $P$. aeruginosa (superinfection) [14], and it has infected both non-CF parents of a CF patient, causing significant morbidity [15]. In addition, there is evidence for greater morbidity amongst CF patients colonised with the LES compared to those carrying non-epidemic strains of $P$. aeruginosa [16].

Recently, we demonstrated that some LES isolates express an unusual phenotype characterized by the early and over-expression of QS-regulated virulence genes, including those encoding the secretion of pyocyanin, LasA and elastase [17]. The unusual phenotype was called hypervirulence because of increased virulence in a Drosophila infection model [17]. Here we refer to it as the over-production (of pyocyanin; OP) phenotype. We reported previously that this phenotype was detected in isolates from both non-CF parents and the CF patient of the unusual infection episode [15] but was lacking from an isolate (LES400) associated with a chronic infection [17].

In this paper, we determine the prevalence of LES isolates exhibiting the OP phenotype amongst adult CF patients in Liverpool, and report a wider analysis of LES isolates with respect to phenotypic and genotypic variations.

\section{Results \\ Efficacy of a simple Luria broth test for pyocyanin production}

In order to test the efficacy of a simple screening procedure for pyocyanin production by strains of $P$. aeruginosa, the amount of pyocyanin in culture supernatants after overnight growth was quantified by measuring the $\mathrm{A}_{695}$ value for 10 environmental strains, 10 strains associated with bacterial keratitis, 29 non-LES CF and 48 LES isolates (Figure 1). Absorbance values for the supernatants of cultures of LES isolates were significantly higher than those for non-LES CF isolates $(p=0.004)$ or non-CF isolates ( $p$ $=0.015)$. When the known LES lasR mutants were removed from this analysis, the significance levels were increased further ( $p<0.001$ and $p=0.003$ respectively).

\section{Prevalence of the OP phenotype}

A total of 34 non-LES (each isolated from a different CF patient) and 55 LES isolates (isolated from 16 different CF patients and confirmed as LES by PFGE and/or PS21 PCR assay) of $P$. aeruginosa were screened for over-production of pigment by using the simple broth test. Overall, $51 \%$ of the LES isolates produced an increased blue/green coloration (above that of strain PA01) but 49\% had normal or lower than normal pigment production. All of the nonLES isolates fell into the latter category. We selected all potential LES pyocyanin over-producers, and a selection of the other LES isolates, for further study using the full pyocyanin production assay. A wide range of pyocyanin production was observed (Figure 2). We grouped these activities as either (i) OP (pyocyanin $\mathrm{A}_{695}>0.1$ at cell density $A_{600}=1.2$ ); (ii) Intermediate (pyocyanin $A_{695}>0.1$ at cell density $A_{600}=1.7$ ); (iii) Normal (pyocyanin detectable, similar to strain PA01) or Negative (no detectable pyocyanin activity). Using these criteria the LES isolates analysed could be separated according to pyocyanin production into $49 \%$ OP $(n=20), 17 \%$ Intermediate $(n=7)$, $34 \%$ Normal or Negative $(n=14)$. We did not sub-divide the latter two categories because of the difficulty in assigning those strains that did not reach equivalent cell densities to strain PA01. There was considerable variation in the amount of time taken for cultures of the different isolates to reach equal cell densities. Of the $20 \mathrm{CF}$ patients from whom 1-6 isolates were subjected to study, 13 carried at least one OP isolate, and a further four carried at least one isolate of "intermediate" phenotype. For three CF patients, the isolates tested all had either a normal or a negative pyocyanin phenotype. We also screened six nonLES CF isolates using the full pyocyanin production assay. All fell into the categories Normal or Negative (data not shown). 


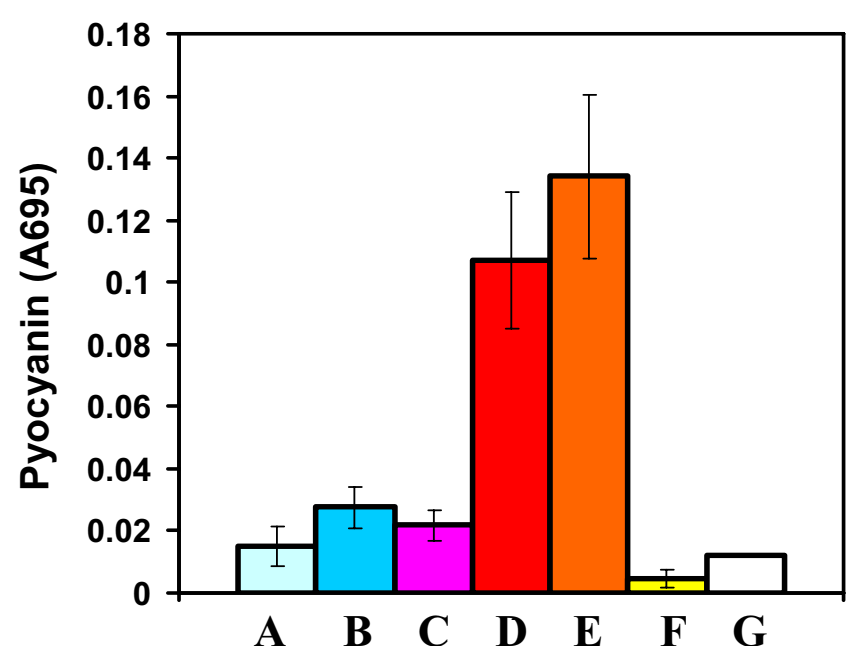

Figure I

Pyocyanin production in culture supernatants after overnight growth in Luria broth. Mean absorbance values are shown for isolates of $P$. aeruginosa falling into the following categories: (a) water isolates $(n=10)$; (b) keratitis isolates $(n=10)$; (c) non-LES CF isolates $(n=29)$; (d) all LES isolates $(n=48)$; (e) LES isolates omitting known las $R$ mutants ( $\mathrm{n}=38)$; ( $\mathrm{f}$ LES known lasR mutants ( $\mathrm{n}=10)$; $(\mathrm{g})$ strain PAOI.

\section{Variations in the OP phenotypes/genotypes amongst multiple isolates from individual patients}

We identified six CF patients (patient A-F) from whom we had archived, multiple and sequential isolates of the LES (1995-2004). For each patient there was a minimum of four different LES isolates and three different sampling dates. There was considerable variation in the colony morphologies of the panel isolates. For each of these isolates both the pyocyanin activity and the LasA activity were assayed (Table 1). In addition, we determined the antimicrobial susceptibilities and, for the majority of isolates, assigned them to a LES pulsotype using PFGE (Table 1). There were widespread variations in both pulsotype and antimicrobial susceptibility data between isolates taken from the same patients.

We tested the panel of isolates for the common mutant phenotypes auxotrophy, lack of swimming motility and hypermutability. Six of the isolates (B55, B67, B41, 109, 49194, B48), from four different patients, exhibited the hypermutable phenotype. Only three isolates were unable to grow on glucose M9 media. These were all isolated from patient B (B46, 49194, B48) and two of the three were hypermutable isolates. All of the panel isolates were non-motile.

Variations in pyocyanin production against cell density for LES isolates taken from patients A-F are shown in Fig- ures 3 and 4 . There was no general consensus in the results obtained. There was some evidence for temporal loss of the OP phenotype in patients $\mathrm{A}, \mathrm{B}$ and $\mathrm{E}$. The earliest isolate from patient A (from 1995) was of OP phenotype. However, the other isolate with the OP phenotype was isolated in 2000. Isolates from patient A included four identical non-OP lasR mutants, one of which was isolated in 1998. For patient B, early isolates were OP-positive whereas later ones were negative. For patient $\mathrm{E}$, only one of two earliest isolates was OP-positive whereas all other isolates were OP-negative. For both patient $\mathrm{B}$ and patient $\mathrm{E}$, two isolates from the same isolation time had different phenotypes with respect to pyocyanin production and LasA activity. This phenomenon could also be observed in patients C, D and F. However, isolates from both 1996 and 2003 from patient $\mathrm{C}$ had the OP phenotype. A similar longevity can be demonstrated with the data from patient $B$. In patient $\mathrm{D}$, none of the isolates had a clear OP phenotype, whereas in patient $\mathrm{F}$, the earliest isolate was non-OP whereas the most recent was OP-positive.

\section{Correlation between the OP phenotype and antimicrobial susceptibilities}

Table 2 summarises the distribution of the OP-positive, OP-negative and other LES isolates according to resistance or susceptibility to five antimicrobial agents. According to the $\phi^{2}$ test for statistical significance, in a comparison between isolates that were OP-positive and other isolates, there was a correlation between the presence of the OP phenotype and resistance to either ceftazidime $(p=$ $0.006)$, aztreonam $(p=0.044)$ or meropenem $(p=0.037)$ (Table 2).

\section{lasR mutations are associated with loss of pyocyanin production}

We analysed 17 of the pyocyanin-negative isolates for mutations in the lasR gene. Of these, lasR mutations were identified in 11 isolates (Table 3). As controls we also sequenced the las $R$ gene from five pyocyanin producers and confirmed that none were mutated (Table 3 ). Thus las $R$ mutations were significantly more frequent amongst pyocyanin-negative isolates $\left(\phi^{2}\right.$ test, $\left.p=0.011\right)$. All of the las $R$ mutations that we have found in LES isolates to date are summarized in Table 3.

\section{Discussion}

We have demonstrated that an unusual phenotype, characterised by early- and over-production of QS-regulated virulence factors such as the exoproducts pyocyanin and LasA protease, is widespread amongst CF isolates of the LES. In a simple broth test for pyocyanin production, whereby cultures were incubated overnight in $5 \mathrm{ml}$ cultures, the levels of pyocyanin were lower than those measured during exponential growth in $50 \mathrm{ml}$ cultures. It is likely that this was either due to some breakdown of pyo- 


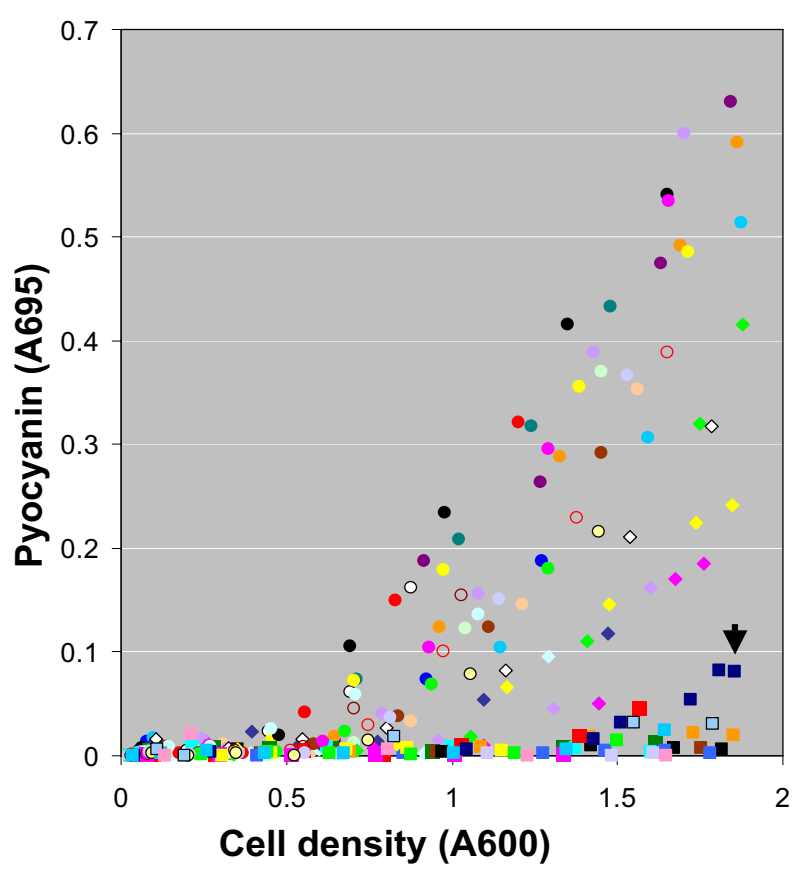

\section{Figure 2}

Pyocyanin assays on LES isolates. Production of pyocyanin in culture supernatants during growth of LES isolates in L-broth is shown for all the LES isolates tested in this study. Each combination of colour and shape plotted represents a different LES isolate. The only non-LES isolate included is strain PAOI (blue squares, indicated by the arrow). LES isolates could be sub-divided on the basis of pyocyanin assays into various groups: (i) OP (early- and over-production of pyocyanin) [circles]. (ii) Intermediate [diamonds]. (iii) Normal or negative [squares]

cyanin or because of greater aeration during growth of the $50 \mathrm{ml}$ cultures in $250 \mathrm{ml}$ flasks. Hence, although the simple broth test is a useful screening tool, the full assay gives a better indication of actual levels of pyocyanin production.

A recent study sought to assess the genetic adaptation of $P$. aeruginosa strains during $\mathrm{CF}$ infections by whole genome analysis of sequential isolates [18]. The authors concluded that virulence factors required for initiation of acute infections are often selected against during chronic infection. Amongst those mutations leading to the loss of virulenceassociated phenotypes, lasR mutations were the most common [18]. Indeed, the observation that lasR mutants are common amongst clinical and environmental isolates of $P$. aeruginosa has led to the suggestion that the QS system may have a negative impact on the organism's fitness
[19]. If this is the case, then the unusual OP phenotype of the LES might be expected to amplify any negative impact. However, we found evidence that the OP phenotype can persist amongst isolates within a patient for periods in excess of five years (patients A, B and C) and up to seven years (patients $\mathrm{B}$ and $\mathrm{C}$ ). To our knowledge there are no other reported instances of natural isolates of $P$. aeruginosa that exhibit the OP phenotype. Given that the OP phenotype places a considerable metabolic burden on the bacteria, the retention of such a high-maintenance phenotype within LES populations suggests that there may be some selective advantage. The fact that the OP phenotype is retained increases the possibility that it may play a role in the transmissibility of the strain. Interestingly, we also found that the same lasR mutant can persist within a patient for up to six years (patient A). We did not find complete correlation between pyocyanin over-production and high levels of LasA activity. This suggests that secondary mutations not affecting the whole QS regulon also lead to diverse phenotypes in LES populations.

$\mathrm{CF}$ isolates are often non-motile or auxotrophic. In one study, 33\% of CF patients had a mean percentage auxotroph count of more than $50 \%$ total $\mathrm{cfu} / \mathrm{ml}$ [20]. Oliver et al. [21] reported an overall prevalence for the hypermutable phenotype amongst CF isolates of $P$. aeruginosa as $20 \%$. In our study, we found that LES isolates exhibiting the hypermutable phenotype comprised $18 \%$ of the isolates from the six CF patients. It has been suggested that the hypermutator phenotype plays an important role in the development of multiple-antimicrobial resistance in $P$. aeruginosa strains causing chronic lung infections [22]. However, we found little evidence that hypermutable isolates were less susceptible to antimicrobials than other non-hypermutable isolates from the same patient (Table 1).

Variations in colony morphology are also commonplace. Normal laboratory practice when dealing with sputum samples is to select a limited number of the most numerous colony morphotypes for storage, antimicrobial susceptibity profiling and occasionally strain typing. Morphology has been linked to increased virulence in the case of small colony variants (SCV) [23]. However, it has also been demonstrated that identical colony morphotypes sharing the same genotype can vary in activities such as antimicrobial susceptibility [24]. In our study, isolates obtained from an individual patient at the same sampling time differed in morphology, but there are a number of examples with sequential isolates where the same colony morphotype and genotype exhibited variations in antimicrobial susceptibity and/or QS-regulated activities. If pulsotype variation is discounted there are further examples suggesting that colony morphotype is an imperfect basis for the selection of isolate variants (Table 1). It has already 
Table I: Phenotypes and genotypes of sequential LES isolates from six CF patients

\begin{tabular}{|c|c|c|c|c|c|c|c|c|c|c|c|c|}
\hline Patient & Year & Isolate & PFGE & Pyocyanin (OP phenotype) & LasA $\{$ SD $\}$ & lasR mut. & CAZ & MER & ATM & TOB & CIP & Colony morphology \\
\hline A & 1995 & B7 & I-II & + & $24.2\{4.9\}$ & NO & $\mathrm{R}$ & $\mathrm{R}$ & $\mathrm{R}$ & $\mathrm{D}$ & $\mathrm{D}$ & SCV,NM,Ra,S \\
\hline A & 1998 & B32 & $|-| \mid$ & - & $98.4\{7.2\}$ & YES & $\mathrm{S}$ & $\mathrm{R}$ & $\mathrm{S}$ & $\mathrm{D}$ & $S$ & SCV,NM,F,S \\
\hline A & 2000 & B45 & ID-II & - & $67.3\{1.9\}$ & nd & $\mathrm{R}$ & $\mathrm{R}$ & $\mathrm{R}$ & $\mathrm{R}$ & $\mathrm{S}$ & NM,F,S \\
\hline A & 2003 & B25 & $\mathrm{I}-\mathrm{II}$ & - & $98.6\{0.6\}$ & YES & $\mathrm{S}$ & $\mathrm{R}$ & $\mathrm{S}$ & $\mathrm{S}$ & $S$ & $\mathrm{NM}, \mathrm{Ra}, \mathrm{S}$ \\
\hline A & 2003 & B70 & IC-II & - & $82.7\{5.1\}$ & NO & $\mathrm{R}$ & $\mathrm{R}$ & $\mathrm{R}$ & $\mathrm{S}$ & $S$ & $\mathrm{M}, \mathrm{Ra}, \mathrm{S}$ \\
\hline A & 2004 & 49111 & ND & - & $98.7\{1.6\}$ & YES & $\mathrm{S}$ & $\mathrm{R}$ & $\mathrm{S}$ & $S$ & $S$ & $\mathrm{NM}, \mathrm{Ra}, \mathrm{RW}$ \\
\hline A & 2004 & 49074 & ND & - & $97.0\{1.5\}$ & YES & $\mathrm{R}$ & $\mathrm{R}$ & $\mathrm{R}$ & $\mathrm{S}$ & $S$ & $\mathrm{NM}, \mathrm{Ra}, \mathrm{S}$ \\
\hline B & 1996 & B46 & IH-IIE & + & $81.6\{2.2\}$ & NO & $\mathrm{R}$ & $\mathrm{R}$ & $\mathrm{R}$ & $D$ & $\mathrm{D}$ & SCV,NM,F,S \\
\hline B & 1998 & B38 & $|-| \mid$ & + & $84.8\{2.9\}$ & NO & $\mathrm{R}$ & $\mathrm{R}$ & $\mathrm{R}$ & $D$ & $S$ & $\mathrm{NM}, \mathrm{Ra}, \mathrm{S}$ \\
\hline B & 2003 & $\mathrm{~B} 60$ & IE-II & + & $20.6\{1.9\}$ & nd & $\mathrm{R}$ & $\mathrm{R}$ & $\mathrm{R}$ & $\mathrm{D}$ & $S$ & NM,F,S \\
\hline B & 2003 & B48 & $|-| \mid$ & - & $93.2\{0.9\}$ & NO & $S$ & $\mathrm{R}$ & $\mathrm{R}$ & $\mathrm{S}$ & $S$ & $\mathrm{M}, \mathrm{Ra}, \mathrm{S}$ \\
\hline B & 2004 & 49194 & ND & - & $93.6\{0.9\}$ & NO & $S$ & $\mathrm{~S}$ & $\mathrm{~S}$ & $S$ & $\mathrm{R}$ & $\mathrm{NM}, \mathrm{Ra}, \mathrm{S}$ \\
\hline C & 1996 & B49 & IC-II & + & $43.3\{4.4\}$ & nd & $\mathrm{R}$ & $\mathrm{R}$ & S & $S$ & $S$ & SCV,NM,F,S \\
\hline C & 1998 & B40 & $\mathrm{I}-\mathrm{II}$ & + & $20.3\{2.3\}$ & nd & $\mathrm{S}$ & $\mathrm{R}$ & S & $\mathrm{R}$ & $S$ & SCV,NM,F,S \\
\hline C & 1998 & B4I & I-II & - & $98.6\{1.3\}$ & YES & $S$ & $\mathrm{R}$ & S & $\mathrm{D}$ & $S$ & $\mathrm{NM}, \mathrm{Ra}, \mathrm{S}$ \\
\hline C & 1998 & 109 & ND & + & $82.7\{1.1\}$ & nd & $S$ & $\mathrm{~S}$ & S & $\mathrm{S}$ & $\mathrm{S}$ & NM,F,S \\
\hline C & 2003 & B64 & $\mathrm{I}-\mathrm{II}$ & - & $95.2\{1.8\}$ & NO & $\mathrm{S}$ & $\mathrm{S}$ & S & $\mathrm{S}$ & $\mathrm{D}$ & SCV,NM,F.S \\
\hline C & 2003 & B65 & I-II & + & $8.61\{7.8\}$ & nd & $\mathrm{S}$ & $\mathrm{R}$ & $S$ & $\mathrm{D}$ & $S$ & $\mathrm{NM}, \mathrm{Ra}, \mathrm{S}$ \\
\hline C & 2004 & 49137 & ND & INT & $63.4\{3.4\}$ & nd & $\mathrm{S}$ & $\mathrm{S}$ & S & $\mathrm{D}$ & $\mathrm{R}$ & SCV,NM,F,S \\
\hline$D$ & 1996 & B28 & IC-II & - & $41.3\{5.5\}$ & NO & $S$ & $\mathrm{R}$ & $S$ & $\mathrm{~S}$ & $\mathrm{~S}$ & SCV,NM,F,S \\
\hline$D$ & 1998 & B35 & IC-II & INT & $96.6\{1.2\}$ & NO & $\mathrm{S}$ & $\mathrm{S}$ & S & $\mathrm{D}$ & $\mathrm{S}$ & SCV,NM,F,S \\
\hline$D$ & 2002 & B6I & IC-II & NOR & $3.33\{4.4\}$ & nd & $\mathrm{R}$ & $\mathrm{R}$ & $\mathrm{S}$ & $\mathrm{R}$ & $\mathrm{D}$ & $\mathrm{NM}, \mathrm{Ra}, \mathrm{S}$ \\
\hline D & 2002 & B62 & I-IIG & - & $95.3\{5.4\}$ & YES & $\mathrm{S}$ & $\mathrm{S}$ & $\mathrm{S}$ & $\mathrm{S}$ & $\mathrm{S}$ & SCV,NM,F,S \\
\hline$D$ & 2002 & B63 & I-II & - & $98.5\{1.9\}$ & YES & $\mathrm{S}$ & $\mathrm{R}$ & $\mathrm{S}$ & $\mathrm{R}$ & $\mathrm{S}$ & $\mathrm{NM}, \mathrm{Ra}, \mathrm{S}$ \\
\hline$E$ & 1995 & B4 & IC-II & - & $95.2\{2.0\}$ & YES & $\mathrm{S}$ & $S$ & $\mathrm{~S}$ & $\mathrm{R}$ & $S$ & $\mathrm{NM}, \mathrm{Ra}, \mathrm{S}$ \\
\hline$E$ & 1995 & B57 & I-II & + & $26.8\{3.2\}$ & NO & $\mathrm{R}$ & $\mathrm{R}$ & $\mathrm{R}$ & $\mathrm{S}$ & $\mathrm{D}$ & $\mathrm{NM}, \mathrm{Ra}, \mathrm{S}$ \\
\hline$E$ & 2000 & B50 & I-II & - & $96.4\{3.6\}$ & NO & $\mathrm{R}$ & $\mathrm{R}$ & $\mathrm{R}$ & $\mathrm{S}$ & $S$ & $\mathrm{M}, \mathrm{Ra}, \mathrm{S}$ \\
\hline$E$ & 2000 & B5I & I-II & - & $98.4\{2.6\}$ & YES & $\mathrm{S}$ & $\mathrm{S}$ & $\mathrm{S}$ & $\mathrm{R}$ & $\mathrm{S}$ & SCV,NM,Ra,S \\
\hline$E$ & 2002 & B67 & ID-IIB & - & $98.4\{2.9\}$ & YES & $\mathrm{R}$ & $\mathrm{R}$ & $\mathrm{R}$ & $S$ & $\mathrm{D}$ & NM,F,S \\
\hline$F$ & 1996 & B30 & I-II & - & $98.5\{1.0\}$ & YES & $\mathrm{S}$ & $\mathrm{S}$ & $\mathrm{R}$ & $\mathrm{R}$ & $\mathrm{S}$ & SCV,NM,Ra,S \\
\hline$F$ & 2000 & B55 & I-II & NOR & $34.8\{4.4\}$ & nd & $\mathrm{S}$ & $\mathrm{S}$ & $\mathrm{S}$ & $\mathrm{S}$ & $\mathrm{S}$ & $\mathrm{M}, \mathrm{Ra}, \mathrm{S}$ \\
\hline$F$ & 2002 & B68 & IA-II & NOR & $88.5\{4.4\}$ & nd & $\mathrm{S}$ & $\mathrm{S}$ & $\mathrm{S}$ & $\mathrm{S}$ & $S$ & NM,F,S \\
\hline $\mathrm{F}$ & 2002 & B69 & IB-IID & + & $31.6\{5.6\}$ & nd & $\mathrm{R}$ & $\mathrm{R}$ & $\mathrm{R}$ & $\mathrm{R}$ & $S$ & NM,F,S \\
\hline
\end{tabular}

PFGE refers to pulsotypes obtained with Spel and Xbal respectively; LasA activity indicates the percentage of the initial (time zero) $A_{600}$ value remaining after 60 min; SD: standard deviation for four replicates; lasR mut. refers to whether the isolate is a confirmed las $R$ mutant or not by sequencing; ND: not determined; antimicrobial agent abbreviations are ceftazidime (CAZ), aztreonam (ATM), meropenem (MER), tobramycin (TOB) and ciprofloxacin (CIP); colony morphology was scored as mucoid (M), nonmucoid (NM), flat $(F)$ raised $(\mathrm{Ra})$, smooth $(\mathrm{S})$, rough/wrinkled $(\mathrm{RW})$, small colony variant (SCV). For OP phenotype: + indicates OP-positive; - indicates OP-negative; INT indicates intermediate; NOR indicates normal.

been reported that the antibiotic susceptibilities of LES isolates vary widely $[12,17]$.

The LES was first identified in a CF unit where ceftazidime monotherapy was being used as standard antipseudomonal treatment for respiratory exacerbations [11]. The authors suggested that the strategy of monotherapy had contributed to the success of the ceftazidime-resistant LES. Blázquez and colleagues [25] have not only suggested that ceftazidime concentrations thought previously to be lethal are not, but that ceftazidime treatment can increase the frequency at which $P$. aeruginosa mutates. Thus, it is entirely plausible that the strategy of ceftazidime therapy may have contributed to the emergence of the OP phenotype exhibited by the LES.

Although first identified because of the spread of a multiresistant clone in a CF unit [11], it is clear that isolates of the LES vary considerably in their antimicrobial susceptibility profiles. We found an interesting correlation between the OP phenotype and resistance to the $\beta$ - lactams ceftazidime, aztreonam and meropenem. It has been demonstrated recently that pyocyanin can act as a signalling molecule in $P$. aeruginosa strains PA01 and PA14, upregulating in late exponential or stationary phase, a limited number of genes from the QS network, including the mexGHI-opmD genes involved in efflux, redox processes and iron acquisition [26]. We have reported previously that these genes are up-regulated in late exponential phase in the OP-positive isolate LES431 when compared to either an OP-negative LES isolate or strain PA01 [17]. Not only does this highlight another possible consequence of the early and over-production of pyocyanin in LES populations, but it suggests that pyocyanin itself may play a direct role in the observed link between antimicrobial susceptibilities and the OP phenotype.

Our data clearly support the notion that bacterial populations in the sputum samples of CF patients are mixed with respect to both genotype and phenotype, even when isolates represent the same clone. We saw many minor vari- 

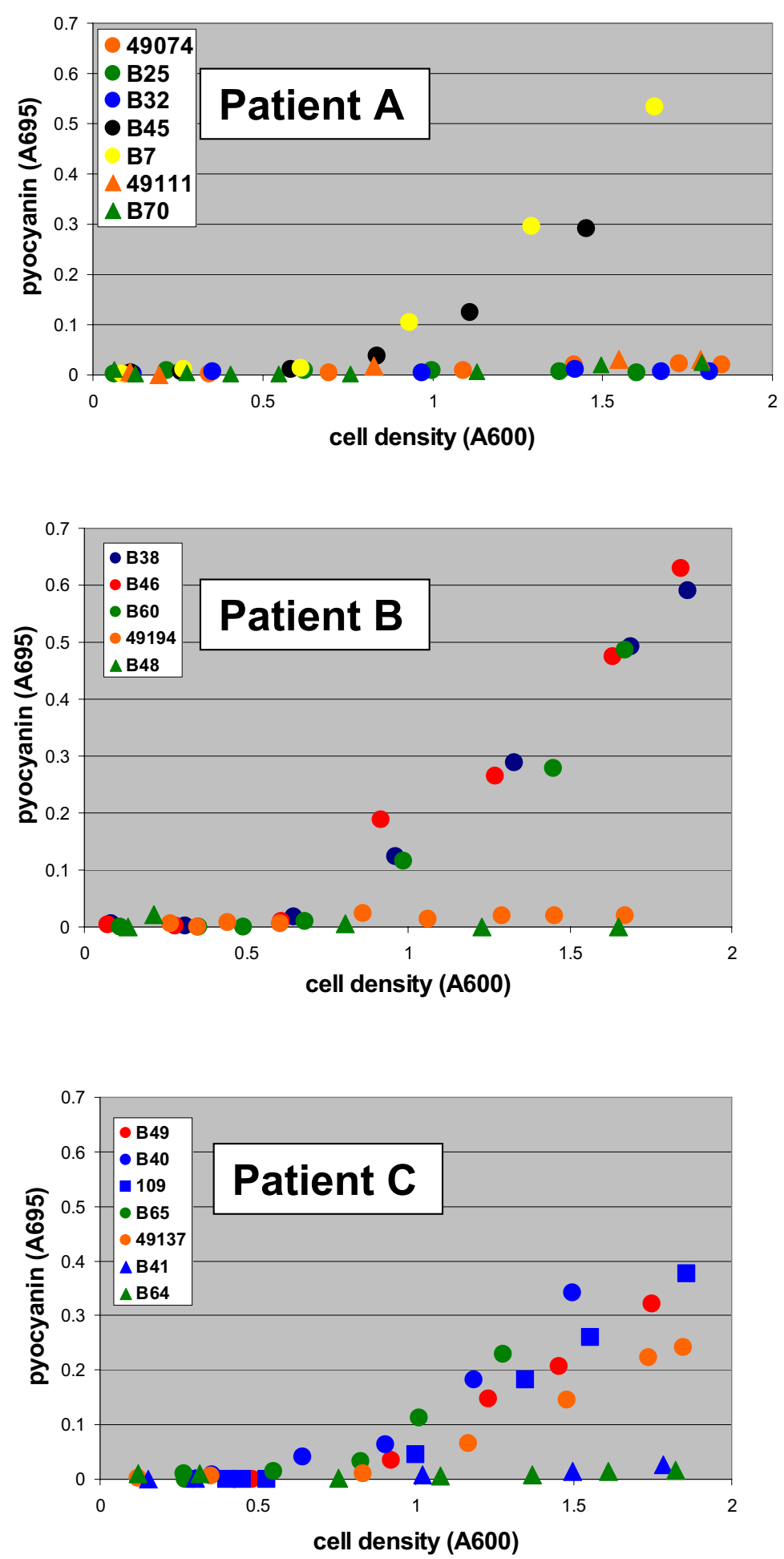

Figure 3

Pyocyanin production by sequential isolates from CF patients A-C. Production of pyocyanin in culture supernatants during growth of LES isolates in L-broth is shown for isolates from three CF patients (patient A-F). Chronological order of isolation is indicated by colour from the earliest to the most recent in the following order : yellow (1995), red (1996), blue (1998), black (2000), green (2003) and orange (2004). Different isolates from the same year are indicated by different shapes. 

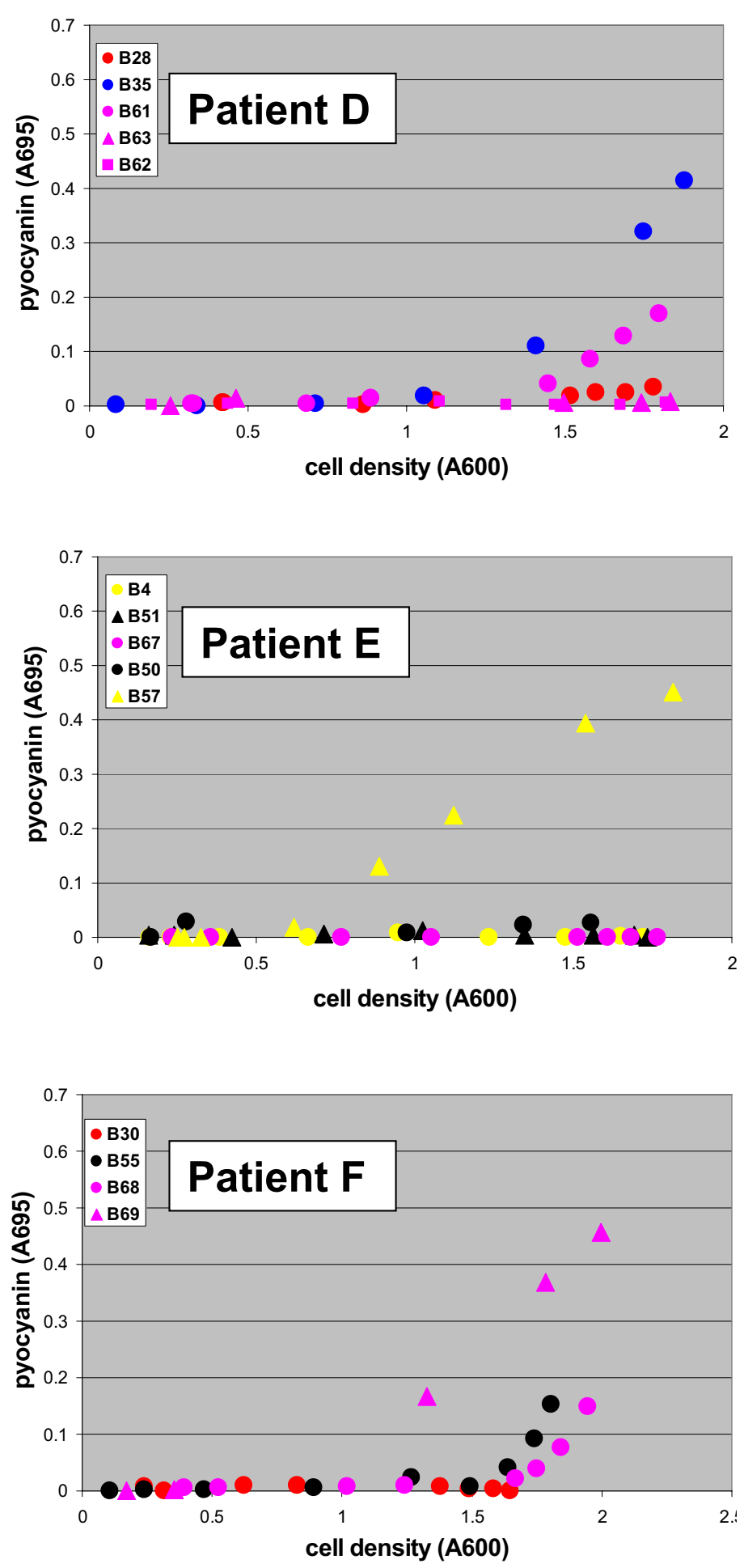

Figure 4

Pyocyanin production by sequential isolates from CF patients D-F. Production of pyocyanin in culture supernatants during growth of LES isolates in L-broth is shown for isolates from three CF patients (patient D-F). Chronological order of isolation is indicated by colour from the earliest to the most recent in the following order : yellow (1995), red (1996), blue (1998), black (2000) and pink (2002). Different isolates from the same year are indicated by different shapes. 
Table 2: Comparison between antimicrobial susceptibility and OP phenotype of LES isolates

\begin{tabular}{lccc}
\hline Antimicrobial agent & \multicolumn{3}{c}{ \% resistant LES isolates for different OP phenotypes } \\
\cline { 2 - 4 } & OP $(n=11)$ & Negative $(n=17)$ & N or I $(n=5)$ \\
CAZ R & 73 & 24 & 20 \\
ATM R & 64 & 35 & 0 \\
MER R & 91 & 65 & 20 \\
TOB R & 27 & 24 & 20 \\
TOB D & 45 & 12 & 40 \\
CIP R & 0 & 6 & 20 \\
CIP D & 27 & 12 & 20 \\
\hline
\end{tabular}

CAZ, ceftazidime; ATM, aztreonam; MER, meropenem; TOB, tobramycin; CIP, ciprofloxacin; R, resistant; D, intermediate antimicrobial susceptibility; N, normal; I, intermediate OP phenotype

ations in PFGE banding patterns between isolates of the LES, even between isolates taken from the same sputum sample. The LES seems to have an unstable genotype $[13,27]$, suggesting that individuals within LES populations undergo deletions, insertions and rearrangements. As well as these quite large genetic events, there are often point mutations leading to significant phenotypic changes. A study analysing greater numbers of isolates from each sputum sample may give a far better insight into the diversity within populations of $P$. aeruginosa in CF than the snapshot obtained when analysing relatively small numbers.

\section{Conclusion}

Isolates of a CF epidemic strain of $P$. aeruginosa exhibit considerable phenotypic and genotypic diversity. The OP phenotype, characterized by de-regulation of the QS system leading to increased production of exoproducts including pyocyanin, is common amongst LES isolates and can persist within CF patients for several years. The presence and widespread nature of such an unusual phenotype amongst populations of a highly successful CF epidemic strain, suggests that the OP phenotype may play an important role in the success of the LES.

\section{Methods \\ Bacterial strains}

The bacterial strains used in this study were mainly isolates of $P$. aeruginosa from adults and children attending CF centres in Liverpool between 1995 and 2004. 34 nonLES isolates were obtained from 21 male and 13 female adult CF patients. The 55 LES isolates were obtained from 10 male and six female CF patients and included isolates from both adults and children over 14 years old. Of the LES isolates, 33 were from patients colonised with the LES for $>4$ years whereas 22 were from patients colonised

Table 3: LES lasR mutants

\begin{tabular}{|c|c|c|}
\hline $\begin{array}{l}\text { LES } \\
\text { isolate }\end{array}$ & lasR Mutation & Comments/Reference \\
\hline LESB4I & deletion of CCGGGCTGGCTACG leading to divergence from position $\mathrm{R}_{66}$ onward & isolated from patient $C$ \\
\hline LESB30 & single point mutation leading to divergence from position $\mathrm{S}_{33}$ onward & isolated from patient $F$ \\
\hline LESB32 & single nucleotide insertion/frame-shift mutation leading to divergence from position $\mathrm{L}_{223}$ onward & isolated from patient $A$ \\
\hline LESB25 & same as LESB32 & isolated from patient $A$ \\
\hline LES49III & same as LESB32 & isolated from patient $A$ \\
\hline LES49074 & same as LESB32 & isolated from patient $A$ \\
\hline LESB63 & same as LESB32 & isolated from patient $D$ \\
\hline LESB62 & single point mutation leading to single amino acid change $\mathrm{H}_{224} \rightarrow \mathrm{R}$ & isolated from patient $D$ \\
\hline LESB4 & same as LESB32 & isolated from patient $E$ \\
\hline LESB5 I & same as LESB32 & isolated from patient $\mathrm{E}$ \\
\hline LESB67 & deletion of $A A G C G G A A$ leading to divergence from position $A_{134}$ onward & isolated from patient $E$ \\
\hline LES49220 & single point mutation leading to single amino acid change $G_{235} \rightarrow D$ & CF isolate, isolated in 2004 \\
\hline LES400 & repetition of GGTGCTC leading to divergence from $\mathrm{G}_{123}$ onward & {$[17]$} \\
\hline LES430 & deletion of GTGGATGCTC leading to divergence from position $W_{152}$ onwards & {$[17]$} \\
\hline LESB44 & insertion of GAAG leading to divergence from position $I_{35}$ onwards & {$[17]$} \\
\hline
\end{tabular}


with the LES for $<2$ years, including 15 from first-time LES-positive cultures. Similar data was not available for the non-LES isolates because strain-specific identification is carried out routinely only for the LES.

Ten $P$. aeruginosa strains associated with bacterial keratitis [28] and ten environmental water isolates [29] were used to assess levels of pyocyanin production by non-CF isolates. All strains were stored in glycerol-Luria broth at $80^{\circ} \mathrm{C}$ and cultured on Luria agar at $37^{\circ} \mathrm{C}$. Strain PA01 [30] was used as a control.

\section{Phenotypic tests}

Auxotrophy was tested by determining the ability to grow on glucose M9 minimal media. Swimming motility was assessed by inoculating bacteria on to the surface of $0.3 \%$ $(\mathrm{w} / \mathrm{v})$ agar plates containing $1 \%(\mathrm{w} / \mathrm{v})$ tryptone and $0.5 \%$ $(\mathrm{w} / \mathrm{v}) \mathrm{NaCl}$. The diameter of the swimming zone was measured after incubation at $37^{\circ} \mathrm{C}$ for $24 \mathrm{~h}$. Each isolate was tested in triplicate. Hypermutability was assessed by determining spontaneous mutation rates on Luria agar containing rifampicin $(300 \mu \mathrm{g} / \mathrm{ml})$ following overnight growth in Luria broth as described previously [21]. Colony morphology was observed on MacConkey agar plates.

\section{Typing by pulsed-field gel electrophoresis (PFGE) and PS2 I PCR assay}

PFGE of SpeI- or XbaI-digested genomic DNA was carried out using $1 \%(\mathrm{w} / \mathrm{v})$ agarose gels. Isolates with identical banding patterns were considered to be genetically indistinguishable and isolates with banding patterns differing by three or fewer bands to be clonal variants (pulsotypes) of the same strain. The PS21 PCR assay for identification of the LES has been described previously [31].

\section{Assays for QS-regulated phenotypes}

Pyocyanin and LasA protease assays were carried out on culture supernatants during growth in Luria broth as described previously [17]. The amount of pyocyanin in culture supernatants was quantified by measuring the $\mathrm{A}_{695}$ value. For overnight cultures, it was important to shake the culture vigorously before obtaining the supernatant. This is to ensure that all of the pyocyanin present is in the oxidized form [32]. LasA protease activity was measured by determining the ability of $P$. aeruginosa culture supernatants to lyse boiled Staphylococcus aureus cells leading to a decrease in $\mathrm{A}_{600}$. Activity was expressed as the percentage of the initial (time zero) $A_{600}$ value remaining after 60 min.

We used a simple broth test to identify strains with increased blue/green pigment production compared to strain PA01. Following overnight growth in universals containing $5 \mathrm{ml}$ Luria broth, with shaking at 200 RPM, cultures were scored as either (i) greater pigment produc- tion than strain PA01 or (ii) similar or less pigment production than strain PA01. For validation of the test, pyocyanin production, as measured by $\mathrm{A}_{695}$ values in culture supernatants, was compared for various categories of $P$. aeruginosa isolates. Statistical significance was tested by using a one way ANOVA test (MINITAB 14.0, Minitab Inc.). Full pyocyanin production assays were carried out using $250 \mathrm{ml}$ flasks containg $50 \mathrm{ml}$ Luria broth.

\section{Identification of lasR mutations}

PCR amplification was carried out as described previously [31]. Amplification of the las region was carried out using the oligonucleotide primer sets lasRF (5'- GTGCCGAATCCATATTTG-3')/lasRR (5'-CCTTCCCTATATATCTGC-3') and lasF1 (5'- GGGTATTCAGTTCGCATA -3')/lasR1 5'CCCAAATTAACGGCCATA-3') to amplify overlapping regions which can be combined to cover the whole lasR gene. PCR amplicons were purified using S-400 microspin columns (Amersham-Pharmacia Biotech) and sequenced by Lark Technologies Inc. using the same oligonucleotide primers employed in the PCR amplification.

\section{Antimicrobial susceptibility tests}

Antimicrobial susceptibility was assessed by disk diffusion tests according to BSAC guidelines [33] using disks (Oxoid) containing ceftazidime $(30 \mu \mathrm{g})$, meropenem (10 $\mu \mathrm{g})$, aztreonam $(30 \mu \mathrm{g})$, tobramycin $(10 \mu \mathrm{g})$ and ciprofloxacin $(5 \mu \mathrm{g})$. Breakpoints for sensitive $(\mathrm{S})$, intermediate (D) and resistant (R) organisms were according to BSAC guidelines.

\section{Authors' contributions}

JLF and SP carried out most of the laboratory work leading to data acquisition. CAH, MJW and TLP participated in the design, supervision and coordination of the study, and in revision of the manuscript. CW participated in the design, supervision and coordination of the study, and drafted the manuscript. All authors read and approved the final manuscript.

\section{Acknowledgements}

This work was undertaken under the framework of the UK CF Microbiology Consortium [34], an initiative funded by the Big Lottery Fund in association with the Cystic Fibrosis Trust. P. aeruginosa water isolates were a kind gift from Dr. Jean-Paul Pirnay, Queen Astrid Military Hospital, Belgium.

\section{References}

I. Latifi A, Winson MK, Foglino M, Bycroft BW, Stewart GS, Lazdunski A, Williams P: Multiple homologues of LuxR and LuxI control expression of virulence determinants and secondary metabolites through quorum sensing in Pseudomonas aeruginosa PAO I. Mol Microbiol 1995, 17:333-343.

2. Ran H, Hassett DJ, Lau GW: Human targets of Pseudomonas aeruginosa pyocyanin. Proc Natl Acad Sci U S A 2003, 100: |43|5-| 4320.

3. Lau GW, Hassett DJ, Ran H, Kong F: The role of pyocyanin in Pseudomonas aeruginosa infection. Trends Mol Med 2004, 10:599-606. 
4. Wilson R, Pitt T, Taylor G, Watson D, MacDermot J, Sykes D, Roberts D, Cole P: Pyocyanin and I-hydroxyphenazine produced by Pseudomonas aeruginosa inhibit the beating of human respiratory cilia in vitro. J Clin Invest 1987, 79:22I-229.

5. Kanthakumar K, Taylor G, Tsang KW, Cundell DR, Rutman A, Smith S, Jeffery PK, Cole PJ, Wilson R: Mechanisms of action of Pseudomonas aeruginosa pyocyanin on human ciliary beat in vitro. Infect Immun 1993, 6 I:2848-2853.

6. Denning GM, lyer SS, Reszka KJ, O'Malley Y, Rasmussen GT, Britigan BE: Phenazine-I-carboxylic acid, a secondary metabolite of Pseudomonas aeruginosa, alters expression of immunomodulatory proteins by human airway epithelial cells. Am J Physiol Lung Cell Mol Physiol 2003, 285:L584-L592.

7. Look DC, Stoll LL, Romig SA, Humlicek A, Britigan BE, Denning GM: Pyocyanin and its precursor phenazine-I-carboxylic acid increase IL-8 and intercellular adhesion molecule-I expression in human airway epithelial cells by oxidant-dependent mechanisms. J Immunol 2005, I 75:40I7-4023.

8. Usher LR, Lawson RA, Geary I, Taylor CJ, Bingle CD, Taylor GW, Whyte MK: Induction of neutrophil apoptosis by the Pseudomonas aeruginosa exotoxin pyocyanin: a potential mechanism of persistent infection. J Immunol 2002, 168:186I-I868.

9. Allen L, Dockrell DH, Pattery T, Lee DG, Cornelis P, Hellewell PG, Whyte MK: Pyocyanin production by Pseudomonas aeruginosa induces neutrophil apoptosis and impairs neutrophil-mediated host defenses in vivo. I Immunol 2005, I 74:3643-3649.

10. Kong F, Young L, Chen Y, Ran H, Meyers M, Joseph P, Cho YH, Hassett DJ, Lau GW: Pseudomonas aeruginosa pyocyanin inactivates lung epithelial vaculoar ATPase-dependent cystic fibrosis transmembrane conductance regulator expression and localization. Cell Microbiol 2006, 8: I I II-I I33.

II. Cheng K, Smyth RL, Govan JR, Doherty C, Winstanley C, Denning N, Heaf DP, van Saene H, Hart CA: Spread of beta-lactam-resistant Pseudomonas aeruginosa in a cystic fibrosis clinic. Lancet 1996, 348:639-642.

12. Panagea S, Winstanley C, Parsons YN, Walshaw MJ, Ledson MJ, Hart CA: PCR-based detection of a cystic fibrosis epidemic strain of Pseudomonas Aeruginosa. Mol Diagn 2003, 7:195-200.

13. Scott FW, Pitt TL: Identification and characterization of transmissible Pseudomonas aeruginosa strains in cystic fibrosis patients in England and Wales. J Med Microbiol 2004, 53:609-615.

14. McCallum SJ, Corkill J, Gallagher M, Ledson MJ, Hart CA, Walshaw MJ: Superinfection with a transmissible strain of Pseudomonas aeruginosa in adults with cystic fibrosis chronically colonised by $\mathbf{P}$ aeruginosa. Lancet $200 \mathrm{I}, 358: 558-560$.

15. McCallum SJ, Gallagher MJ, Corkill JE, Hart CA, Ledson MJ, Walshaw MJ: Spread of an epidemic Pseudomonas aeruginosa strain from a patient with cystic fibrosis (CF) to non-CF relatives. Thorax 2002, 57:559-560.

16. Al Aloul M, Crawley J, Winstanley C, Hart CA, Ledson MJ, Walshaw MJ: Increased morbidity associated with chronic infection by an epidemic Pseudomonas aeruginosa strain in CF patients. Thorax 2004, 59:334-336.

17. Salunkhe P, Smart CH, Morgan JA, Panagea S, Walshaw MJ, Hart CA, Geffers R, Tummler B, Winstanley C: A cystic fibrosis epidemic strain of Pseudomonas aeruginosa displays enhanced virulence and antimicrobial resistance. J Bacteriol 2005, I 87:4908-4920.

18. Smith EE, Buckley DG, Wu Z, Saenphimmachak C, Hoffman LR, D'Argenio DA, Miller SI, Ramsey BW, Speert DP, Moskowitz SM, Burns JL, Kaul R, Olson MV: From the Cover: Genetic adaptation by Pseudomonas aeruginosa to the airways of cystic fibrosis patients. Proc Natl Acad Sci U S A 2006, 103:8487-8492.

19. Heurlier K, Denervaud V, Haas D: Impact of quorum sensing on fitness of Pseudomonas aeruginosa. Int J Med Microbiol 2006, 296:93-102

20. Thomas SR, Ray A, Hodson ME, Pitt TL: Increased sputum amino acid concentrations and auxotrophy of Pseudomonas aeruginosa in severe cystic fibrosis lung disease. Thorax 2000 55:795-797.

21. Oliver A, Canton R, Campo P, Baquero F, Blazquez J: High frequency of hypermutable Pseudomonas aeruginosa in cystic fibrosis lung infection. Science 2000, 288: $|25|-\mid 254$.

22. Macia MD, Blanquer D, Togores B, Sauleda J, Perez JL, Oliver A: Hypermutation is a key factor in development of multipleantimicrobial resistance in Pseudomonas aeruginosa strains causing chronic lung infections. Antimicrob Agents Chemother 2005, 49:3382-3386.

23. von Gotz F, Haussler S, Jordan D, Saravanamuthu SS, Wehmhoner D, Strussmann A, Lauber J, Attree I, Buer J, Tummler B, Steinmetz I: Expression analysis of a highly adherent and cytotoxic small colony variant of Pseudomonas aeruginosa isolated from a lung of a patient with cystic fibrosis. J Bacteriol 2004, 186:3837-3847.

24. Foweraker JE, Laughton CR, Brown DF, Bilton D: Phenotypic variability of Pseudomonas aeruginosa in sputa from patients with acute infective exacerbation of cystic fibrosis and its impact on the validity of antimicrobial susceptibility testing. J Antimicrob Chemother 2005, 55:921-927.

25. Blazquez J, Gomez-Gomez JM, Oliver A, Juan C, Kapur V, Martin S: PBP3 inhibition elicits adaptive responses in Pseudomonas aeruginosa. Mol Microbiol 2006.

26. Dietrich LE, Price-Whelan A, Petersen A, Whiteley M, Newman DK: The phenazine pyocyanin is a terminal signalling factor in the quorum sensing network of Pseudomonas aeruginosa. Mol Microbiol 2006.

27. Smart CH, Walshaw MJ, Hart CA, Winstanley C: Use of suppression subtractive hybridization to examine the accessory genome of the Liverpool cystic fibrosis epidemic strain of Pseudomonas aeruginosa. J Med Microbiol 2006, 55:677-688.

28. Winstanley C, Kaye SB, Neal TJ, Chilton HJ, Miksch S, Hart CA: Genotypic and phenotypic characteristics of Pseudomonas aeruginosa isolates associated with ulcerative keratitis. J Med Microbiol 2005, 54:519-526.

29. Pirnay JP, Matthijs S, Colak H, Chablain P, Bilocq F, Van Eldere J, De Vos D, Zizi M, Triest L, Cornelis P: Global Pseudomonas aeruginosa biodiversity as reflected in a Belgian river. Environ Microbiol 2005, 7:969-980.

30. Stover CK, Pham XQ, Erwin AL, Mizoguchi SD, Warrener P, Hickey M], Brinkman FS, Hufnagle WO, Kowalik DJ, Lagrou M, Garber RL, Goltry L, Tolentino E, Westbrock-Wadman S, Yuan Y, Brody LL, Coulter SN, Folger KR, Kas A, Larbig K, Lim R, Smith K, Spencer D, Wong GK, Wu Z, Paulsen IT, Reizer J, Saier MH, Hancock RE, Lory $\mathrm{S}$, Olson MV: Complete genome sequence of Pseudomonas aeruginosa PAOI, an opportunistic pathogen. Nature 2000, 406:959-964.

31. Parsons YN, Panagea S, Smart CH, Walshaw MJ, Hart CA, Winstanley C: Use of subtractive hybridization to identify a diagnostic probe for a cystic fibrosis epidemic strain of Pseudomonas aeruginosa. J Clin Microbiol 2002, 40:4607-46II.

32. Hernandez ME, Newman DK: Extracellular electron transfer. Cell Mol Life Sci 200I, 58:I562-I57I.

33. Andrews JM: The development of the BSAC standardized method of disc diffusion testing. J Antimicrob Chemother 200I, 48 Suppl I:29-42.

34. UK CF Microbiology Consortium [http://www.cfmicrobiol ogy.org.uk/]
Publish with BioMed Central and every scientist can read your work free of charge

"BioMed Central will be the most significant development for disseminating the results of biomedical research in our lifetime. "

Sir Paul Nurse, Cancer Research UK

Your research papers will be:

- available free of charge to the entire biomedical community

- peer reviewed and published immediately upon acceptance

- cited in PubMed and archived on PubMed Central

- yours - you keep the copyright
BioMedcentral 